\title{
Narrative Ethik am Beispiel des Romans Never Let Me Go von Kazuo Ishiguro und weitere Überlegungen
}

\author{
Dietmar Mieth
}

\begin{abstract}
„Der Mensch ist bestimmt, den Menschen neu zu schaffen.“ (meiner Erinnerung nach ein Spruch am Eingang des amerikanischen Nationalmuseums)
\end{abstract}

\section{Einleitung}

Als jahrzehntelanger Grenzgänger zwischen normativer Wissenschaftsethik und literarisch orientierter narrativer Ethik versuche ich im Folgenden zunächst, das dialektische Verhältnis von Fakt und Fiktion in Science, Arts und ,Literatur' ana$\log \mathrm{zu}$ betrachten. Analogie bedeutet, dass das Verschiedene das Ähnliche zwar überwiegt, aber nicht aufhebt. Ich beginne mit einer Erzähltheorie auf anthropologischer Basis, um eine übergreifende Perspektive zu gewinnen. Auf dieser Grundlage betrachte ich das Verhältnis zwischen empirischer Rationalitätskontrolle und Fiktion in der Biomedizin. An dem literarischen Beispiel, dem Roman Never Let Me Go (2005) von Kazuo Ishiguro versuche ich, eine narrative Entfiktionalisierung wissenschaftlicher Fiktionen zu zeigen. Sie wird als Methode u. a. dazu angewandt, den Diskurs um Zukunftsvisionen, die um das Klonen von Menschen und um den Organersatz kreisen, mit emotionaler Betroffenheit anzureichern um damit Fragen der Verantwortung zu provozieren.

Im zweiten Teil dieser Untersuchung greife ich ein Thema auf, das sich dem ersten Zugriff der Interpretation auf den Roman Never Let Me Go zu entziehen scheint: die Unsicherheit, die den Leser durch eine bestimmten Art des Erzählens erfasst. Diese Art des Erzählens schränkt die Perspektive der erzählenden Person - genau wie in dem Roman, den ich als Beispiel heranziehe - so ein, dass sie ,unzuverlässig' erscheint. Der Autor zieht sich gegenüber dem Erzähler oder der Erzählerin so zurück, dass man ihm nicht richtig auf die Spur kommt. Daraus entsteht die Uneindeutigkeit selbst einer plausiblen Interpretation. Die dabei entstehende Ungewissheit erlaubt es, sich ganz mit dem emotionalen Klima, das die Erzählung erzeugt, zufrieden zu geben, aber auch, diesem Stachel der Ungewissheit nachzugehen. Dies versuche ich im zweiten Teil, anhand narratologischer Überlegungen zu erläutern. ${ }^{1}$

1 Ein interessantes Beispiel für die Erzeugung von Unsicherheit bieten die Erzählungen der Nobelpreisträgerin Alice M. Munro, siehe z. B. in der Sammlung Der Traum meiner Mutter (2006). 


\section{Eine anthropologische Theorie des Erzäblens}

A narrative text is a text in which an agent relates (,tells $\left.s^{\circ}\right)$ a story in a particular medium, such as language, imagery, sound, buildings, or a combination of thereof. A story is a fabula that is presented in a certain manner. A fabula is a series of logically and chronologically related events that are caused or experienced by actors. An event is the transition from one state to another state. Actors are agents that perform actions. They are not necessarily human. To act is defined here as a cause or to experience an event. The assertion that a narrative text is one in which a story is related implies that the text is not identical to the story. (Bal 1997: 5)

Ich greife aus diesem Zitat die doppelte und von mir hervorgehobene Erwähnung von Erfahrung, experience, heraus. Im Englischen ist der Unterschied zwischen Erleben und Erfahren nicht markiert. In meiner Untersuchung über Moral und Erfahrung (Mieth 1999) berücksichtige ich diese Unterscheidung, d. h. wie sich nach meiner Ansicht Erleben zu Erfahren erschließt und dabei den literarischen Erzählvorgang präjudiziert. Literarisches Erzählen basiert auf der Erfahrung komplexer Vorgänge und versucht, für diese eine Form, eine künstlerische Gestaltung zu finden. Diese Vorgänge unterliegen empirischen, aber auch experientiellen Formen ihrer Erfassung. Sie sind zudem in abrufbare Wissensspeicher eingeordnet. Sie lassen experimentelle Überprüfungen zu.

Das Erfassen vollzieht sich über das Erinnern. Im Erinnern werden Fakten, Erlebnisse und Wissensdaten angeeignet, repräsentiert und transformiert. Die Transformation geschieht zunächst, indem die Erinnerung sich selbst formt, indem sie sich selbst erzählt. (Das Selbst ist hier zugleich Subjekt und Objekt.) Erzählen kann nach Mieke Bal, in Wortform, aber auch in Form von Bildern oder von Tönen erfolgen (5). Das Sich-Selbst-Erzählte lässt sich weiter erzählen. Dabei unterliegt das Erzählen in der Literatur Entwicklungen, Konventionen und deren Brüchen, Konterkarierungen, Selbstaufhebungen und dergleichen.

Mit diesen Bemerkungen habe ich mich nicht in literarische Erzähltheorien einmischen wollen. Es genügt mir, Erzählen als anthropologische Konstante mit der ethischen Selbstreflexion zu verbinden. Literarisches Erzählen kann zwar ohne moralische Bevormundung von außen ihre Form und Gestalt finden, enthält aber auch eine zumindest evokative Seite für die Selbstbefragung, an die sich wiederum die Frage nach Gut und Böse, nach Richtig und Falsch, anschließt. Darüber hinaus: wie erzählt wird, ist für die Autoren auch eine moralische Angelegenheit. Dazu sagt Paul Ricœur:

Dass die narrative Funktion nicht ohne ethische Implikationen ist, legt bereits die Verwurzelung der literarischen in der mündlichen Erzählung nahe. [...] Man mag einwenden, dass die literarische Erzählung auf der Ebene der eigentlich narrativen Gestaltung diese ethischen Bestimmungen zugunsten der rein ästhetischen Bestimmungen verliert. Hiermit würde man sich über das Ästhetische selbst täuschen. Sicherlich impliziert die Lust, die wir verspüren, wenn wir das Schicksal einer Figur verfolgen, dass wir jegliches eigentlich moralische Urteil - im gleichen Augenblick, in dem wir die tatsächliche Handlung außer Kraft setzen - suspendieren. Aber im irrealen Bereich der Fiktion erforschen 
wir unablässig neue Bewertungsweisen für Handlungen und Figuren. Die Gedankenexperimente, die wir im großen Laboratorium der Einbildung durchführen, sind auch Forschungsreisen durch das Reich des Guten und des Bösen. Etwas umzuwerten, möglicherweise sogar abzuwerten bedeutet immer noch, es zu bewerten. Das moralische Urteil ist nicht abgeschafft, es ist vielmehr selbst den der Fiktion eigenen imaginativen Variationen unterstellt. Dank dieser Bewertungsübungen im Bereich der Fiktion kann die Erzählung letzten Endes ihre Erschließungs- und Verwandlungsfunktion gegenüber dem Empfinden und Handeln des Lesers in der Phase der Refiguration der Handlung durch die Erzählung ausüben. (Ricœur 1996: 201)

Paul Ricœur betrachtet die Begegnung mit Literatur - auch er denkt an erzählende Literatur - als einen schöpferischen Prozess der Aneignung und Transformation. Man versteht diesen am besten, wenn man einen moralinteressierten und begründungskompetenten Leser unterstellt, der der Literatur nicht passiv begegnet, sondern diese mit seinen moralrelevanten Einsichten bewusst konfrontiert. Für dieses Verfahren schlägt Ricœur einen Dreischritt für die Analyse des Verarbeitungspotentials von und mit Literatur vor: die Präfiguration, die Konfiguration und die Refiguration. Dieser Dreischritt enthält Elemente der alten Mimesisthese in subjektiver Wendung, insofern eine differenzierte Aneignung und eine schöpferische, subjektaufgeladene ,Nachahmung' erfolgt.

In der Präfiguration kann der analytische und konstruktive Leser in einer Art hermeneutischen Akt sein Vorverstehen ablösen und testen. Lesen ist in diesem Sinne Selbstaufklärung über das, was dem Lesen vorausliegt, aber eben als Vorausliegendes durch das Lesen erst bewusst wird. Man erkennt sozusagen, mit welcher Brille man liest, etwa schon daran, wo man Leseschwierigkeiten hat. In der Konfiguration wird das unvermeidliche Mitgehen mit den Figuren, die Sympathieverteilung, die Teilidentifikation, der Abstand bis zum Abscheu als reflektierte und konfrontierte Erfahrung präsent. Da diese Figurationen kompetente Leistungen des interpretierenden Lesers sind, enthalten sie zugleich ein ,draußen und ein ,drinnen', Nähe und Distanz in wechselseitiger Bedingung des Verwandelns von Leseerlebnis in Selbsterfahrung. Insofern tritt der Schritt der Refiguration zwar aus der Begegnung heraus, erzählt sie sich erinnernd zugleich wiederum selbst und gibt ihr dadurch eine bleibende Präsenz als ein moralrelevanter Bewusstseinsgehalt. Freilich ist damit nur eine sehr formale Zuschreibung von moralischer Relevanz erfolgt. Ist diese Relevanz konstruktiv, indem sie die Entdeckung von Lebensmöglichkeiten enthält, welche ich mit Heinrich Rombach und Theodor W. Adorno „ethische Modelle“ (vgl. Mieth 1983, 1999) genannt habe? Und ist mit diesen Möglichkeiten nicht immer auch die Einsicht in Hindernisse und Unmöglichkeiten gegeben, so dass ihr kritisch destruktives Potential ihren Hinweischarakter übersteigt? Letzteres wird in den Theorien sichtbar, die literarische Erfahrung eher im Modus der Produktion von Ungewissheit sehen.

Die Reflexionen Ricœurs über Zeit und Erzäblung (1988-91) haben vor allem den Begriff der „narrativen Identität“ sowohl für Gemeinschaften als auch für Individuen gebildet (Ricœur 1988-91: III 395 ff.). In einer differenzierten Mime- 
sis-Theorie wird der „Modellcharakter“ in den Erzählformen durch Tradierung, Konfrontation und Selbstfindung beschrieben. Die Frage nach dem Zusammenhang von Identitätsbildung und der Narrativität behandelt Charles Taylor (1996; vgl. Haker 1999: 74-118). Damit verbunden ist auch bei anderen, teils Kommunitaristen, teils Neoaristotelikern, ein Paradigma nicht-normativer Ethik, das zuletzt oft ,Ethik des Strebens' (im Unterschied zur ,Ethik des Sollens') genannt wurde. $^{2}$

Zunächst will ich aber auf die Erzählkomponente in der Wissenschaft als Science eingehen und die Frage beantworten, an welchen Stellen und warum diese Erzählkomponente zwischen facts and fiction einzuordnen ist.

\section{Wissenschaft: empirische Rationalitätskontrolle, Phantasie und Fiktion}

Wenn sich mithin in [...] vielen [...] Fällen der Glaube als diejenige Kraft erweist, die das gesammelte wissenschaftliche Einzelmaterial erst zur richtigen Wirksamkeit bringt, so darf man sogar noch einen Schritt weiter gehen und behaupten, daß schon beim Sammeln des Materials der vorausschauende und vorfühlende Glaube an die tieferen Zusammenhänge gute Dienste leisten kann. Er zeigt den Weg und er schärft die Sinne. Einem [...] Experimentator, der im Laboratorium seine Versuchsanordnung aufbaut und die gemachten Aufnahmen unter die Lupe nimmt, wird in vielen Fällen der Fortschritt der Arbeit [...] erleichtert durch eine gewisse, mehr oder weniger klar bewußte besondere Gedankeneinstellung, mit welcher er seine Untersuchungen einrichtet und die gewonnenen Ergebnisse betrachtet und deutet. (Planck 1959: 248)

An die Stelle des „vorfühlenden Glaubens“ kann man in diesem Zitat auch das vorausentwerfende Urteil oder eine stimulierende Vision zu stellen versuchen. In meinem Beitrag über Wissenschaft und Vorurteil (Mieth 2009) habe ich ganz im Sinne von Gadamers Wabrheit und Methode (Gadamer 1986) darzustellen versucht, dass man ohne Vorausurteile nicht auskommt, diese sich freilich nicht zu Vorurteilen verhärten dürfen. Vorurteile sind an der Korrekturunfähigkeit und an der trotz Widerlegungen fortgesetzten Selbstbehauptung erkennbar.

Moderne Wissenschaft ist mit unerschöpflicher Neugier (vgl. Blumenberg 1980 zur curiositas) und mit Steigerung auf Zukunft hin ausgestattet. WissenschaftTechnik-Ökonomie ist überall dort, wo Wissenschaft nicht bloß erklären will, ein pragmatischer Fortschrittszwang in der Erkenntnis. Wissenschaft muss wegen dieser Einbettung in zukunftsproduzierende Abläufe auch Zukunft erzählen. Ein solcher Erzählablauf ist zum Beispiel die Patentierung, in der es zugleich um Erkenntnisneuheit, um Nutzungsplausibilität und um Privilegien geht (vgl. das Copyright).

Wissenschaft wird im Zusammenhang mit Wissenschaftsförderung vorauserzählt. Die narrativen facts sind dabei in fließendem Übergang zur narrativen ficti-

2 Dies hat vor allem das Werk von Krämer (1992) bewirkt. Vgl. auch dazu Haker (1999: 11952). 
on. Die Phantasie ist in der Wissenschaft oft vorwärtsgebunden, denn man will ja Fortschritte erzielen. Beispiele:

- Die Atomkraftenergie in den siebziger Jahren: der GAU und das Problem der Endlagerung werden mit (Un-)Wahrscheinlichkeitsrechnungen versehen. Heute ist ein Ausstieg nicht als Nullsumme zu haben: der durch die Institutionalisierung erfolgte Problemzuwachs bleibt erhalten.

- French Anderson und die Gentherapie im Jahr 1986: „Ich weiß es nicht (ob es funktioniert), aber ich glaube daran". ${ }^{3}$

- Die Zuschreibung von Tod und Lebensanfang: man sucht für einen moralischen ,Status' einen empirischen ,Status' (Hirntod, Status des Embryos). Das erscheint wie ein pragmatischer Zirkel: die Praxis bestimmt die Reflexion im Hinblick auf ihre Selbstbehauptung. Das Problem der Zuschreibung eines moralischen Status beim Menschen hat Eilert Herms gründlich untersucht (vgl. Herms 2008: 108). Er kritisiert u. a. protestantische Ethiker, die sich auf diese Zuschreibung eingelassen haben, obwohl doch, seiner Ansicht nach, Menschsein aus theologischen Gründen nicht vom Menschen selbst als Status eines buman being definiert, sondern nur aufgrund der Gegebenheit eines solchen Lebewesens respektiert werden müsste. Ich füge hinzu, dass auch der Ausdruck „Anerkennung“ (Honneth 2012) ein problematisches Element der Zuschreibung enthält. Theologisch wäre davon die „unbedingte Annahme“ (Haker 2001: 224) zu unterscheiden.

- „Therapeutisches Klonen“ - Da es keine absehbare Therapie, sondern nur eine Intention gibt, impliziert der Begriff eine sich objektiv gebende Werbesprache, die sich bis in kirchliche Dokumente einschleicht (vgl. Mieth 2001: 63-73).

- Die grüne Gentechnik wird von den Problemen der unkontrollierbaren Freisetzung, der Resistenzbildung (bei Insekten) und des Reinvestitionszwanges aufgrund der Saatgutabhängigkeit der Farmer begleitet. Senator Luggar kommentierte diese Probleme als Vorsitzender des Senatsausschusses für USamerikanische Landwirtschaft im Jahr 2003: „Wenn Probleme entstehen, werden wir sie lösen". ${ }^{4}$

Nun kann man natürlich sagen, die Wissenschaft muss sich vorwissenschaftlich in der Gesellschaft behaupten, weil die Gesellschaft sie sonst nicht versteht, aber fallen Forschungen im Bereich der genannten Beispiele nicht z. B. unter Wissenschaftsskepsis? Auch die Wissenschaft als ein selbstkritisches und falsifizierungsfähiges Erkenntnis-System steht m. E. unter dem Motto: Man soll Probleme nicht so lösen, dass die Probleme, die aus der Problemlösung entstehen, größer sind als die Pro-

3 Quelle: Der Autor selbst als Teilnehmer einer zur Diskussion von Anderson eingeladenen Expertengruppe katholischer Universitäten. Anderson untersuchte damals Mäuseblut als Vorstufe für die Ermöglichung des Gentransfers. Es lag nahe, ihn auf die Differenz zwischen seinen Versuchen und seinen Versprechungen auf einem Poster hinzuweisen. 
bleme, die gelöst werden. (So meine Fassung des sogenannten principle of caution; vgl. Mieth 2002: 371).

Ich habe Beispiele gewählt, die man drastisch nennen kann. Man könnte sie leicht erweitern (z. B. im Hinblick auf Gendoping). Die ,Wissenschaft', auch die ,Biomedizin', ist natürlich ein viel komplexeres Phänomen. Aber mir kommt es darauf an, zu zeigen, dass zwei fiktionale Elemente zur Wissenschaft zu gehören scheinen: der Vorausentwurf des Paradigmas und die narrative Beschreibung einer praktischen und förderungsfähigen Intention bzw. technisierbaren Innovation, die dann zum Wirtschaftsfaktor wird (vgl. Mieth 2002: 10-21). Wissenschaft ist selbst-narrativ und fremd-narrativ, in beiden Fällen spielen Fiktionen eine Rolle, einlösbare und nicht einlösbare Fiktionen, jedenfalls solche, die überprüfbar und gegebenenfalls rückholbar formuliert werden müssen. Dies kann man das „Transparenzgebot“ nennen (vgl. Beck 2013: 37 ff.). Nur mit der Einhaltung des Transparenzgebotes wird $\mathrm{m}$. E. die Problemlösungsregel effektiv.

Als ich im Beraterteam der Ausstellung Genwelten (in Bonn, Mannheim, Dresden, Genf u. a.) war, wollte ich in der Bundesausstellungshalle in Bonn auf dem Fußboden „Stolpersteine“ zum Nachdenken anbringen lasse. ${ }^{5}$ Stattdessen wurde entschieden: die Fragen der ethischen Verantwortung übernimmt die Kunst. So standen nachher ein paar geklonte Tiere herum. Was also kann die Kunst, wenn es um Verantwortungsfragen geht? Man kann nicht sagen, der zeitgenössische Roman, zum Teil auch der Film (The Clonus Horror, 1979; Gattaca, 1997; Blueprint, 2003; The Island, 2005) verweigere sich der Aufgabe, Nachdenklichkeit zu erzeugen. Der Roman, den ich im Folgenden auf die Frage nach seiner Herstellung von Verantwortungsfragen in der Rezeption hin analysieren werde, wurde ebenfalls unter dem Titel Never Let Me Go verfilmt (Romanek 2010).

\section{Das literarische Beispiel Never Let Me Go}

Damit komme ich zu meinem literarischen Beispiel: Kazuo Ishiguros Never Let Me Go (2005), in der deutsch Übersetzung als Alles, was wir geben mussten (2006) betitelt. Der englische Originaltitel zitiert den Anfang eines Songs, den die Heldin, Kathy, gerne hört. In ihm kommt die Liebessehnsucht vor allem als Sehnsucht nach Geborgenheit zum Ausdruck. Dies lässt sich in viele Richtungen entfalten: Sehnsucht nach der fehlenden Elterngeborgenheit der Heimkinder, Sehnsucht nach dauerhafter Beziehung, auch nach eigenen Kindern. So sagt Kathy:

Egal, worum es in dem Lied wirklich ging, ich hatte meine eigene Version im Kopf, als ich dazu tanzte. Wissen Sie, ich stellte mir vor, dass es von einer Frau erzählte, der man mitgeteilt hat, sie könne keine Kinder bekommen. Aber dann bringt sie doch eines zur

5 Vgl. den Sammelband der Bundesaustellungshalle (Ruhnau 2000). In meinem Beitrag „Ethik und Heuchelei am Beispiel der Biopolitik“ (Mieth 2000b) findet sich auch das oben genannte Beispiel der Sprachpolitik in Bezug auf das Klonen. 
Welt und ist unheimlich glücklich, und sie drückt es ganz fest an die Brust, weil sie furchtbare Angst hat, sie könnten voneinander getrennt werden, und sie flüstert ihm zu, Baby, Baby lass mich niemals los. Natürlich handelt das Lied gar nicht davon, aber das war es, was ich mir damals vorgestellt habe. (Ishiguro 2006: 329)

All das ist für Kathy nicht möglich: sie ist, was wir freilich erst erfahren, nachdem wir Sympathie mit der Figur entwickelt haben, ein Klon. Nach dem Roman ist sie damit nicht fortpflanzungsfähig. Kathy ist, wie die anderen Insassen des Internates Hailsham, dazu bestimmt, in Operationen vier Mal Organe zu spenden', wobei das vierte Mal den Tod einschließt. So wird das aber nicht gesagt. Ausgedrückt wird das so: sie haben nun ,abgeschlossen'. Dies wiederum wird im deutschen Titel $\mathrm{Al}$ les, was wir geben mussten angedeutet. Die Heimleiterin, die Kathy bei ihrem kindlichen Tanz beobachtet, fängt an zu weinen und kommentiert dies später:

Ich sah eine neue Welt unaufhaltsam auf uns zukommen. Eine wissenschaftlichere, effizientere Welt, ja. Neue Behandlungsmethoden für die alten Krankheiten. Alles sehr gut. Aber eine harte, grausame Welt. Und ich sah ein kleines Mädchen, das mit fest geschlossenen Augen die freundliche alte Welt an die Brust drückte, eine Welt, die, das wusste sie in ihrem Herzen, nicht bleiben konnte, aber sie hielt sie fest und flehte sie an, sie niemals loszulassen. (330)

So viel zum Titel - zu der in diesem Zitat enthaltenen Nostalgiewelle, die etwas anderes ist als Kathys Sehnsuchtstanz, komme ich später.

Wie immer in der narrativen Ethik, wie ich sie verstehe, suche ich den Zugang über die literarische Form des Erzählens, nicht direkt über den Inhalt. Der Roman wählt einen erzählerischen Trick, um sich nicht in die warnenden Utopien der science fiction als Genre einzureihen. Dieses Genre beherrscht z. B. ein Autor wie Stanisław Lem, wenn er in science fiction-Novellen die Biotisierung der Materien und die Materialisierung des Lebens beschreibt. Das nennt er einmal die „omnigenerative Kreationistik“, der schließlich die Kirchen mit einer Ausweitung des Schöpfungsauftrages dogmatisch zustimmen (Lem 1998; vgl. Mieth 1985). In diese Richtung geht jedoch der Roman nicht, indem er die Szene im Vortext räumlich und zeitlich fixiert: England, am Ende des 20. Jahrhunderts.

Nun wissen wir als Leser gleich zweierlei: Erstens, Ende des 20. Jahrhunderts beherrschte man eine Klon-Methode zur Herstellung von menschlichen Ersatzteil-Entitäten (,Organsäcke') ebenso wenig wie sie heute im Jahre 2014 beherrschbar ist und wie sie möglicherweise nie beherrschbar sein wird. (Es gibt auch in der Gentechnik inzwischen ,Ausstiege'.)

Zweitens: Das, was als eine geographisch und historisch verortete Angelegenheit nacherzählt zu werden scheint, hat es ebenso wenig gegeben wie die Gesetze, die so etwas ermöglicht zu haben scheinen, und die nach dem Roman in Großbritannien beschlossen wurden. Dazu muss freilich eine liberale biomedizinische Gesetzgebung in UK erst narrativ-fiktiv hochgerechnet werden.

Es ist also unserem zeitgenössischen Gefühl nach ganz anders, als es Miss Emily den nachfragenden Helden, Kathy und Tommy, beschreibt: 
Auf einmal eröffneten sich ungeahnte Möglichkeiten, neue Therapien für so viele Krankheiten, die bis dahin als unheilbar galten. Das war es, was die Welt hören wollte und gern zur Kenntnis nahm. Und die längste Zeit zogen es die Leute vor zu glauben, die Organe kämen aus dem Nirgendwo oder wüchsen in einer Art Vakuum heran [...] Wie können sie in einer Welt, die Krebs jetzt für heilbar hält, wie können sie von dieser Welt verlangen, dass sie freiwillig auf die Behandlung verzichtet und in die finsteren Zeiten zurückkehrt? Es gab kein Zurück mehr. [...] deshalb wurden sie [die Klone] lange Zeit totgeschwiegen, und die Leute taten alles, um nicht über sie nachdenken zu müssen. (318 f.)

Indirekt wird unter dem Namen „Morningdale“ mit Hinweis auf „Schottland“ der Wissenschaftler Willmut, der 1997 das Klonschaf Dolly präsentierte, zitiert: „Er wollte werdenden Eltern die Möglichkeit anbieten, Kinder mit verbesserten Eigenschaften zu zeugen" (319 f.). Nach meiner Kenntnis wäre diese Unterstellung besser durch ein Zitat von Robert Edwards, dem nobelpreisgekrönten Miterzeuger des ersten sogenannten ,Retortenbabys' Louise im Jahr 1968, gedeckt - er habe sich, so sagte er 1999, von mir für einen Kongress in Stuttgart eingeladen, um die in vitro-Fertilisation bemüht, denn damit würden seiner Sicht nach Eltern die Verantwortung für die genetische Gesundheit ihrer Kinder wahrnehmen.

Warum entsteht bei der Lektüre Beklemmung, obwohl das von Ishiguro ausgebreitete Szenarium in die Vergangenheit versetzt erscheint? Zunächst dadurch, dass die geographisch-historische Platzierung in der Jetztzeit es nicht von der Erzählung verlangt, Umwelten und Alltage zu verändern. Es muss nicht so viel erfunden werden wie in der science fiction. Es handelt sich um eine wiedererkennbare Welt, die besichtigt werden kann, was die Hauptfiguren am Schluss auch unternehmen, ohne freilich den locus amoenus ihres Internates, das inzwischen gesetzlich nicht mehr erlaubt ist, wiederzufinden. Möglicherweise wird hier mit den auftauchenden und verschwindenden Orten der fiktiven Abenteuerliteratur gespielt. Der Ort heißt Hailsham, wobei hail, englisch auch ,Hagel', im Deutschen leicht andere Assoziationen weckt.

Dass der Roman sich nicht als future fiction, sondern eher als back fiction inszeniert, als erinnerter Rückweg zu einer entschwundenen Welt - die aber dennoch ihre Erzeugnisse, die Klone, die erinnerungsfähig sind, lebend hinterlassen hat ermöglicht zweierlei: Erstens, die Erzählerin Kathy kann mit ihren Mitteln, die offensichtlich Einschränkungen unterliegen, die Ambivalenz zeitgenössisch beschreiben, die ihre eigene Geschichte durchzieht. Zweitens ist nach der im Roman geschilderten Auflösung des Experimentes mit integrierungsfähigen Klonen, die eine ,Seele' haben, die Provokation geklonter menschlicher Ersatzteillager für die Organtransplantation für den Leser nicht aufgelöst, da diese, der Fiktion nach, klinisch abgeschottet, eher der Viehzucht analog, weiter existieren. Es geht „für die Leute“ darum, die Klone als „nicht ganz menschlich“ (319) betrachten zu dürfen. „Sie werden heute“, sagt Miss Emily in ihrem Aufklärungsgespräch für Kathy und Tommy, 
im ganzen Land keine Einrichtung wie Hailsham mehr finden. Alles, was Sie finden, sind diese riesigen staatlichen Heime, die es immer gegeben hat, und selbst wenn sie heute ein bisschen besser sind als früher, glauben sie mir, meine Lieben. Sie würden nächtelang kein Auge zu tun, wenn Sie sähen, wie es in manchen von ihnen immer noch zugeht. (321)

Nicht das Klonen ist verboten, sondern die Eingliederung der Spender-Klone in die Karriereabläufe und in die Bildungsinstitutionen der bürgerlichen Welt. Auf der einen Seite steht der Zwang, der an einem Ausbruchsversuch geschildert wird (6), auf der anderen Seite steht das Experiment Hailsham als eine Fürsorge ohne Aufklärung, die einem moralisch zweideutigen Experiment dient. Zum einen soll gezeigt werden, dass Klone eine ,Seele‘ haben (315). Zum anderen wird dies vor allem auf die künstlerischen Fähigkeiten hin entwickelt, was der Initiatorin, Miss Emily, auch erlaubt, mit den Kunstwerken der Kinder einen Kunsthandel zu führen. Aber eben diese Miss Emily will mit dem Experiment beweisen, dass die Menschenklone, die ihre Herkunft nicht kennen und instrumentell erzeugt sind, zwar sexuelle Beziehungen aufnehmen, sich jedoch nicht fortzeugen können eine sehr fiktive Annahme (siehe oben) -, aber dennoch eine ,Seele', d. h. einen selbstbestimmten Lebensentwurf haben können.

Einer der Lehrerinnen, Miss Lucy, kommt dieses Experiment moralisch problematisch vor. Die biologisch Instrumentalisierten können ja dazu letztlich nicht selbst Stellung beziehen. Miss Lucy versucht, sie aufzuklären und verlässt Hailsham letztlich mit der Einsicht, dass die Betroffenen nicht zu dieser Stellungnahme gelangen können. Was ist die Ursache? Dass sie ,behindert' sind oder dass sie gehindert werden? Miss Lucy rechnet nicht bloß mit einer ästhetisch schöpferischen, sondern mit einer moralisch handlungsfähigen Seele, die aber im Internat um der späteren Verwendung willen, aber auch, weil diese Selbstbestimmung biologisch gehemmt erscheint, nicht ausgebildet wird. So drückt sich die moralische Hilflosigkeit nur in Träumen und Sehnsüchten aus. Die besten Internatsschüler verstehen sich als Künstler, aber ohne eine solche Biographie inszenieren zu können. Oder sie werden, ausgezeichnet mit hohem sozialem Einfühlungsvermögen, „Betreuer“, was bedeutet, dass sie die ihr anvertrauten Lebendspender bei den Spenden begleiten und trösten können. Für diese Aufgaben werden sie nach dem Internat kaserniert, und wenn sie auch lernen, sich in der urbanen Gesellschaft reibungsfrei zu bewegen: sie bleiben irgendwie gehemmt. Diese Einschränkung bringt die Macht der Sehnsüchte umso profilierter hervor, z. B. dann, wenn sie ihre Umwelt nach möglichen Eltern, also den Gametenspendern, aufgrund von Ähnlichkeiten, suchen. Denn dies erscheint als eine Möglichkeit, „einen Blick in die eigene Zukunft werfen“ (170) zu können. Die Zukunftspläne sind jedoch durch die Spendebestimmung eingeschränkt (174 ff.).

Die (biologisch und/oder „moralisch“ eingeschränkte?) Perspektive der Erzählerin verlangsamt und verstärkt zugleich den ausgedrückten Gefühlszustand. In einer Besprechung, abgedruckt auf dem deutschen Klappentext, heißt es: „Ein Meisterwerk, das den Leser gefühlsmäßig auf unerhörte Art sensibilisiert.“ Wer 
den möglichen emotionalen Tiefgang in den Vordergrund stellt, muss andererseits beachten: Gefühlsintensivierung, wie sie dargestellt wird, unterliegt einer gewollten erzählerischen Erzeugung von Unsicherheit. Dahinter steht m. E. durchaus die Frage nach der Verantwortung von eingeschränkter Menschenwürde, verbunden mit der Vorführung von emotionaler Intensität. Geht es dabei um eine folgenlose Nostalgie, wie sie die Heimleiterin erfasst, als sie beim Tanz des kleinen Mädchens zu dem Song „Never Let Me Go“ über eine entschwundene Welt weint? Aber der Autor gilt durchaus als ein Vertreter selbstreflexiver Kunstkritik. Mittels der indirekten Erzählweise durch die Hauptfigur Kathy, die zugleich emotionale Intensität und Hilflosigkeit in der Selbstbestimmung durch eben diese Einschränkung ausstrahlt, gerät der Leser in eine Unsicherheit, ob er sich auf seine begleitenden Urteile verlassen kann. Angesichts der Verunmöglichung einer autonomen Lebensführung geht es im Hintergrund des Romans, aber ohne direkte Benennung dieses Problems, um die fortschreitende Instrumentalisierung des Menschen durch den Menschen. Insofern, um auf die fehlenden Stolpersteine in den Ausstellungen über „Genwelten“ zurückzukommen, hat Ishiguro schon etwas mehr dargestellt als bloße Karikaturen des Fortschritts, auch wenn seine Fiktion die biomedizinischen Fakten, was leicht nachweisbar ist und ihm kaum entgangen sein kann, verletzt.

\section{Narrative Verunsicherung und ibre ethische Bedeutung- eine erweiterte Betrachtung bisheriger Ergebnisse ${ }^{6}$}

Zunächst greife ich auf Ricœur zurück: Die Rede Ricœurs von den „imaginativen Variationen“ der „narrativen Identität“ (1987: 66) enthält sowohl Konsistenz und Kontinuität, als auch Selbsterfindung. Letztere ist in moderner Narration besonders unsicher. Die Ich-Unsicherheit kann auch eine „Entblößung“ sein, die „etwas mit dem ethischen Primat des Anderen als das Selbst gegenüber dem Selbst zu tun hat" (Ricœur 1996: 206). Dann würde weiter gelten: „Indem sie den Charakter narrativisiert, gibt die Erzählung ihm seine Bewegung zurück, die in den erworbenen Dispositionen und den sedimentierten Identifikationen mit verschwunden war" (203). Erzählen erzeugt also eine Freiheit, die aus dem Spiel der Bewertungsexperimente in den Figurationen des Erzählens hervorgeht, die sich aber zugleich auf die in ihr liegende Verbindlichkeit, diese Freiheit zu erhalten und zu gestalten, besinnt. Daraus entsteht jene „Verbindlichkeit des Unverbindlichen" (vgl. Brinkmann 1969), der man sich nicht entziehen kann, weil in ihr die eigene moralische Identität und zugleich für Andere die eigene moralische Identifizierbarkeit zum Vorschein kommt.

Im Roman Never Let Me Go ist das erzählende Selbst besonders unsicher. Kathy ist als Betreuerin so erfolgreich, dass sie sich die Spender, die sie betreut,

6 Vgl. zum Folgenden meine Beiträge zur Ungewissheit und Literaturethik (Mieth 2007a,b). 
selbst aussuchen darf (Ishiguro 2006: 251-64). Aber das ändert nichts an der aus ihrem Anderssein gegenüber der bürgerlichen Karrierewelt erwachsenden Unsicherheit. Kathy hat wie die anderen Hailsham-Klone eine starke soziale Sensibilität. Das ist konstruktiv in Konflikten und Aufregungen, aber zugleich verkompliziert es die persönlichen Beziehungen, denen keine offene Dauer beschert ist. Immer wieder erscheint in der Selbsterzählung die Einschränkung als selbstbestimmtes Handlungssubjekt, die zugleich die innere Szene der Hailshamer verstärkt und ihre Außenbeziehung reduziert. Kathy empfindet die Einsamkeit sehr stark (251 f.). Man fragt sich, ob die Strukturierung der Erzählung durch die Brille der geklonten Kathy nicht auch die Absicht verfolgt, durch diesen ,Trick die scheinbare Eindeutigkeit zu unterlaufen, die ich als eingeschränkte Handlungsfähigkeit, als Mangel an einer autonomen Lebensführung, als Instrumentalisierung, als ethisches Manko markiert habe.

Es ist freilich auch denkbar, dass der Autor diese Angelegenheit diametral anders angegangen ist: er könnte die moralischen Reaktionen auf die Einschränkung der Klone auch ästhetisch eingesetzt haben, um die emotionale Wirkung zu erzeugen, die den Leser in den Roman hineinzieht und in ihm einen ,anderen“ Zustand erzeugt. Das ästhetische Spiel mit der Moral, ja auch mit der ethischen Reflexion ist ein in Literatur und Film durchaus mögliches Verfahren (vgl. Mieth 2006). Im Folgenden versuche ich zu zeigen, wie schwer zu unterscheiden ist, ob es sich um ein ethisches Modell im Sinne eines kritischen Angebotes handelt oder um eine artifiziell konstruierte Verunsicherung, wie man sie auch sonst aus Geschichten kennt (etwa bei den amerikanischen Autoren John Updike und Phillip Roth, die die Eindeutigkeit von Geschlecht und Rassenzugehörigkeit untergraben). ${ }^{7}$

Die Erschütterung von Gewissheiten hat eine lange Tradition. Die Literatur spielt dabei eine gewichtige Rolle, insofern sie nicht Systeme bestätigt, sondern in Frage stellt. Das Leitmotiv des Romans über Tristan und Isolde enthält z. B. eine solche Provokation (Gottfried 2008). Ich habe sie als „Disproportion zwischen Erfahrungsgestalt und Ordnungsgestalt" beschrieben (vgl. Mieth 1983: 238-48). Walter Haug arbeitet in mehreren Beiträgen das „absolute Ethos“ der Tristanminne heraus und damit die nichtintegrierbare radikale Infragestellung der moralischen Gewissheiten (vgl. Haug 2001: 263 f.). Vielleicht kann man allgemein sagen, dass eine Kontrasterfahrung oder gar ein Überdruss an verordneten und abgestumpften Gewissheiten eine Zündung ist, die manches literarische Feuer entfacht. Das will ich an einem Beispiel zeigen.

In Robert Musils bekannter Novelle „Tonka“ (1922), die auch literarische Nachahmer fand, ${ }^{8}$ wird ein literarischer Perspektivismus kultiviert, der Ungewissheit erzeugt. Bekanntlich geht es um ein Verhältnis zwischen einem Ingenieur

7 So beispielsweise in Philip Roths Der Menschliche Makel (2002), bei dem sich ein weißer Professor als Sohn schwarzer Eltern herausstellt.

8 La Dentellière, die Spitzenklöpplerin, von Pascal Lainé als Roman (1974), in dt. Übersetzung (1979) und als Film (1977). 
und einer schwanger werdenden Wäscherin. Die Entstehung der Schwangerschaft erscheint nicht ganz aufgeklärt. Mit dieser fortschreitenden Schwangerschaft schreitet aber zugleich das Unglück des Mädchens, nur schwach durch den Geliebten mit seinen Zweifeln abgefedert, voran. Dabei erscheint die ,einfachere“ Gestalt des Mädchens Tonka als durchsichtig und undurchsichtig zugleich. Das ist der Punkt, der an die Kathy von Ishiguro erinnert. Der Erzähler spricht von der „Fremdheit ihres allzu einfachen Lebens“ (Musil 1922: 103). Das Schlichte ist auch das Komplizierte. ,Denken' und ,Natur' lassen sich nicht harmonisieren. Die Komplexität des reflektierenden Erzählers kehrt sich gegen den Erzähler selbst. Es "erscheint“ ihm etwas, wobei er aber nicht zugleich "dahinter" sieht. Dennoch steht er unter Deutungszwang. Wo er nicht versteht, versucht er, zu erraten. Die Verantwortung leidet unter der Mischung von äußerem Druck (durch die Mutter des Mannes), Scham, Heuchelei und Ungewissheit. Es scheint so, als wenn es eine Gefühlsicherheit („ganz ihm zu gehören“ - Musil 1922) bräuchte, in der sich Tonka, aber nicht ihr Geliebter befindet, um aus dem reflexiven Perspektivismus zu einem richtigen Tun vorzudringen. Die Wahrheit der Person steht gegen die Wahrheit der Argumente. Andererseits ist auch das Gefühl der Ganzheit bei Tonka gesellschaftlich gebrochen bzw. motiviert: gehört man ihm ganz, dann gehört man dazu, gleichsam als bürgerliche Person. Nichts ist also reine Natur, was als ,natürlich' angesehen wird.

Indem die Erzählung die Perspektiven ständig bricht, macht sie aus der einfachen Unglücksgeschichte der mit Kind oder in der Schwangerschaft verlassenen Geliebten, die vom Volkslied über die Operette bis zur Gretchentragödie reicht, ein „Dornengerank“. Die Sehnsucht nach dem „Ganzen“, das mit „Glück“ assoziiert wird (Musil 1922: 67, 78), geht in perspektivischer Unentschlossenheit unter.

\section{Ungervissheit und Täuschung im literarischen Verfabren- John Maxzeell, Coetzees Elizabeth Costello9}

Es ist möglich, dass eine Erzählung den Leser gleichsam durch ihren O-Ton auf einen anderen Weg führt als durch ihren Unterton. Dabei kann freilich zwischen beiden (oder mehr) Tönen eine Art Balance, eine wechselseitige Korrektur, eine Dissonanz entstehen. Dies lässt sich z. B. auch in Bernhard Schlinks Der Vorleser (1995) verfolgen. ${ }^{10}$ Bei Schlink ist dies die Spannung zwischen dem Analphabetentum (die Welt ihrer Scham ohne Schuld) und dem KZ-Aufsehertum (die Welt der Schuld, zunächst ohne Scham) der Protagonistin. Das Lesen lernen führt zu Schuld und Scham, aber auch zur Ausweglosigkeit.

9 Es handelt sich um das Lehrstück 6, das in der fiktiven Erzählung „Das Problem des Bösen“ aus Elizabeth Costello (Coetzee 2003) verhandelt wird. Vgl. dazu Haker (2003).

10 Vgl. dazu Tongeren (2001). 
Coetzee führt in dem Roman Elizabeth Costello (2003) den Leser am Beispiel einer erfundenen selbst erzählenden weiblichen Erfolgsautorin in die Frage der Legitimität von Gewaltdarstellungen ein, welche die gute Absicht der Aufklärung, Dokumentation und Motivation gegen das Unrecht und die Brutalität ins Feld führen. Dennoch führt die Darstellung von Gewalt in einen anderen Zustand und unterstützt eine Reizkultur, der sich der Leser schwer entziehen kann. Die Gewaltdarstellung in der Beschreibung eines real existierenden Autors, Paul West, beschäftigt sich mit den Hinrichtungen der Hitler-Attentäter des 20. Juli 1944. Sie rückt den Tätern, aber auch den Opfern auf den Leib: haben Opfer nicht das Recht auf Diskretion? Ist der Schriftsteller für die Rezeption mit verantwortlich? Muss er seinen impliziten Leser an die Hand nehmen und führen?

Solche und ähnliche Fragen tauchen im erzählenden Kopf der Protagonistin Elizabeth Costello auf. Coetzee hat die Erzählung als Essay-Beitrag zu einer Tagung über Literatur und Gewaltdarstellung (in Berlin) erfunden. Und er hat dabei in der erfundenen Biographie der Elizabeth Costello ein Motiv versteckt, das auch sonst mit unterschiedlichen Funktionen in seinen Romanen auftaucht: eine Vergewaltigung der jungen späteren Autorin durch einen Arbeiter, dem sie in der Romantik einer Emanzipation ,nach unten' für ein sexuelles Erlebnis in sein Domizil gefolgt war. Elizabeth hat diese Geschichte verdrängt, sie lässt sie nicht durch wiederholte Erinnerung und durch Erzählen an sich heran. Dadurch entsteht eine Parallelität zwischen ihrem Motiv der Gewaltverweigerung in der literarischen Darstellung der brutalen Täter-Opfer-Geschichten und ihrem Motiv der Verdrängung. Das Oberthema des Romans wird durch das Unterthema konterkariert, ohne dass die Würfel der Geschichte in eine feste Richtung fallen. Der aufgeklärte Leser kann das zwar nachvollziehen, aber dadurch wird die Geschichte im Hinblick auf ihr scheinbar ,moralisches' Thema antwortlos. Die Geschichte gibt freilich zu denken, kann die Antwort auf die Frage nach der Moral, die jeder mit sich selbst finden muss, präjudizieren, aber nicht determinieren. Aber weil das so ist, wird das Problem eines unzuverlässigen Erzählens diskutiert. Unzuverlässigkeit meint hier: die Erzeugung von Unsicherheit statt z. B. einer klaren ,Moral von der Geschicht‘.

\section{Unzuverlässiges Erzäblen als Methode?}

In seinem Buch Die Rhetorik der Erzäblkunst (1974; englischsprachige Erstauflage The rhetoric of fiction 1961) richtet sich das Interesse von Wayne Booth auf „die rhetorischen Mittel, die dem Autor [...] zur Verfügung stehen, wenn er, bewusst oder unbewusst, versucht, dem Leser seine fiktionale Welt zu suggerieren“ (1974: I 5). Sein Ziel besteht nicht in einem abstrakten Regelwerk, sondern darin, zu zeigen, „was gute Romanautoren effektiv getan haben“ (I 7). Entsprechend diskutiert Booth in seinem Werk hauptsächlich Ansichten bekannter Autoren und allgemeine Grundsätze, die vor allem in der Praxis der Literaturkritik Anwendung finden. Für Booth geht die Leistung herausragender Autoren über ihre Rolle für 
die Erzählung hinaus: „Sie geben dem Leser sicheres Geleit, nicht nur in der Welt der Romane, in denen sie auftreten, sondern auch im Hinblick auf die moralischen Wahrheiten, die in der Welt außerhalb des Buches bestehen“ (I 224).

Booth zufolge strebt jeder Autor Objektivität bzw. Neutralität an, um die Figuren seiner Erzählung möglichst glaubhaft und real zu erschaffen, wozu eine gewisse Neutralität bezüglich ihrer guten und schlechten Eigenschaften notwendig ist. Der schaffende Autor kann es sich demnach nicht erlauben, „seine Vorurteile unmittelbar und unverändert in sein Werk einfließen zu lassen“" (I 77). Er muss seinen Abscheu gegenüber verwerflichen Charaktereigenschaften unterdrücken. Diese zumindest streckenweise Abstraktion des realen Autors von den eigenen Werten beschreibt Booth als das Schaffen eines zweiten Selbst, einer implizierten Version der individuellen Person des Autors. Zwar besteht immer noch eine Korrelation zwischen realem Autor und impliziertem Erzähler, insofern letzterer ein Produkt des ersteren ist, allerdings sind laut Booth die implizierten Erzähler der unterschiedlichen Werke ein und desselben realen Autors nach wie vor zu unterscheiden, da für sie unterschiedliche Wertkonfigurationen denkbar sind.

Mittels des Begriffs des implizierten Erzählers definiert Booth auch einen Erzähler als zu- bzw. unzuverlässig: Er nennt einen Erzähler zuverlässig, der „für die Normen des Werkes (d. h. die Normen des implizierten Autors) eintritt oder in Übereinstimmung mit ihnen handelt, und unzuverlässig, wenn er dies nicht tut“ (I 164). Bei der Klassifizierung eines Erzählers als unzuverlässig geht es Booth also nicht in erster Linie darum, lügende Erzähler von aufrichtigen Erzählern zu unterscheiden und den Wahrheitsgehalt der narrativen Aussagen zu beurteilen. Der eigentliche Reiz unzuverlässigen Erzählens ist vielmehr mit dem Reiz einer ironischen Bemerkung vergleichbar: ${ }^{11}$ Der Leser durchschaut die Unzulänglichkeiten des Erzählers und teilt die Kenntnis des implizierten Autors, „der den Leser bei der Beurteilung des Erzählers für sich gewinnt“ (I 163). Als Beispiel nennt Booth Huckleberry Finn: „der Erzähler behauptet, von Natur aus böse zu sein, während der Autor heimlich hinter seinem Rücken seine Tugenden preist“ (I 164). Unzuverlässiges Erzählen findet also dort statt, wo zwischen impliziertem Autor und Leser ein heimliches Einverständnis besteht (vgl. II 124). Der Leser erkennt ein Defizit in den Ausführungen des Erzählers, ${ }^{12}$ zugleich aber auch eine weitere Möglichkeit, seine Aussagen zu bewerten. Auch wenn dies (gerade auch im Beispiel Huckleberry Finn) nicht zwangsläufig einen Unterschied hinsichtlich der erzählten Tatsachen bedeuten muss, besteht darin ein besonderer, zusätzlicher Genuss für den Leser.

Booth räumt durchaus die Gefahr ein, dass dieses Einverständnis durch die Möglichkeit bedroht ist, dass dem Leser die verborgene Sichtweise des implizier-

11 In seiner „Galerie unzuverlässiger Erzähler und Reflektoren“ spricht Booth explizit von einer „als Unzuverlässigkeit“ apostrophierten „Art von Ironie“ (Booth 1974: II 166).

12 Im Beispiel Huckleberry Finn geht es darum, dass der Erzähler zwar nicht ,böse` ist, es aber trotzdem von sich behauptet. 
ten Autors verschlossen bleibt, allerdings geht Booth in seinem Textverständnis grundsätzlich davon aus, dass das „Urteil des Autors immer gegenwärtig [ist], immer evident für jeden, der danach zu suchen versteht" (I 29).

Diese Theorie Booths ist aus zwei Gründen kritisiert worden: Zum einen wird ihr vorgeworfen, bei der $\mathrm{Zu}$ - bzw. Unzuverlässigkeit gehe es quasi ausschließlich um moralische Werturteile des Erzählers. Ferner gebe es keine stringente Methode, mittels derer sich der Status des implizierten Autors, vor allem dessen Wertvorstellungen, ermitteln ließe. So formuliert beispielsweise Ansgar Nünning den „Verdacht, dass der implied author als Sammelbecken für all jene Aspekte dient, die die Erzähltheorie nicht ohne weiteres im Rahmen eines kohärenten Modells integrieren kann“ (Nünning 1998: 14). Gérard Genette meint: „Meines Erachtens muss die Narratologie nicht über die narrative Instanz hinausgehen, und die Instanzen des, implied author ${ }^{6}$ und des, implied reader ' liegen nun einmal jenseits von ihr“" (Genette 1998: 284). Genette verortet das Phänomen des „implied author" allgemein in der Poetik (284). Demnach ist ein implizierter Autor „ein Bild des (realen) Autors, das der Text konstruiert und das der Leser als solches wahrnimmt“, was wiederum der „Lektüreerfahrung“ entspreche (286).

Ansgar Nünning bemüht sich, eine kognitiv-narratologische Theorie unzuverlässigen Erzählens mittels einer frame theory zu beschreiben, in deren Zentrum textuelle Signale und kontextuelle Parameter stehen. Die gängigsten Kennzeichen unzuverlässigen Erzählens bestünden im Vorhandensein homodiegetischer Erzähler, die nicht nur in die erzählte Geschichte involviert sind, sondern auch als „konkret fassbare, personalisierbare Sprecher auf der Ebene der erzählerischen Vermittlung in Erscheinung treten" (Nünning 1998: 6), die das Geschehen kommentieren und oftmals offensichtliche Defizite aufweisen. Das trifft auf die Erzählerin von Never Let $M e$ Go zu. Darüber hinaus nennt Nünning als wichtigstes Kriterium, dass eine Diskrepanz zwischen der Darstellung des Erzählers und „einer zweiten Version des Geschehens, derer sich der Erzähler nicht bewusst ist und die sich Rezipienten durch implizite Zusatzinformationen erschließen können" (6) besteht. Auch dies konnten wir im Hinblick auf Ishiguros Roman zeigen. Erkennbar ist diese Konstitution auch an der impliziten Selbstcharakterisierung des Erzählers. Der Wirkungseffekt von unzuverlässigem Erzählen liegt demnach für Nünning „darin, die Aufmerksamkeit des Rezipienten von der Ebene des Geschehens auf den Sprecher zu verlagern“ (19). Führt also Ishiguro mehr in die auf den Leseprozess beschränkte Identifikation mit der Hauptperson Kathy hinein, oder will er ,zuverlässig' mehr aussagen lassen? Nünning spricht von einer Methode des Lesens, durch die Wiedersprüche innerhalb des Textes und zwischen der fiktiven Welt des Textes und dem Wirklichkeitsmodell des Lesers aufgelöst werden (5). Letztendlich bedeutet dies, dass „ein Erzähler nicht an sich unglaubwürdig ,ist', sondern dass es sich dabei um eine Feststellung des Betrachters handelt, die historisch, kulturell und letztlich sogar individuell stark variieren kann" (25). Im Text würden bestimmte Signale gesetzt, durch die Reaktio- 
nen des Lesers ausgelöst werden. Dabei spielen - das habe ich gezeigt - außertextuelle Faktoren eine Rolle, auf die der Leser beim Lesen bewusst oder unbewusst Bezug nimmt, beispielsweise ein Werte- und Normensystem, aber auch schon allgemeines Weltwissen. Also gilt es, zwischen den textuellen Signalen (beispielsweise explizite Widersprüche zwischen Aussagen des Erzählers oder der konkrete Hinweis des Erzählers auf Einschränkungen, denen er unterliegt) und dem herangezogenen Bezugsrahmen (beispielsweise historische Fakten oder dem Genre des Textes, in unserem Fall die Fakten der Biomedizin) zu unterscheiden. Das ist freilich Aufgabe des Interpreten, eine Aufgabe, die man nicht auf jeden Leser ausdehnen kann. Dennoch kann sich auch der Leser der Frage nach seiner Identität nicht leicht entziehen.

Der Literaturwissenschaftler Harold Bloom hat darauf hingewiesen, dass das Lesen fiktionaler Texte, bei dem man sich in andere Menschen hineinversetzt, dabei hilft, einen eigenen, ,autonomen' Standpunkt zu gewinnen (vgl. Bloom 1991). Dies geschieht nicht durch Nachahmung, sondern durch Auflösung von Vorurteilen, Traditionen, Umwelten, Konventionen. Diese werden durch fiktionale Erzählungen durchschaubar und bis zu einem gewissen Grad kontrollierbar.

Richard Rorty folgt diesem Konzept insofern nicht ganz, als er die Loslösung von diesen (All-)Gemeinheiten nicht für wirklich möglich hält (vgl. Rorty 2003). Aber auch dieser Skeptiker hält die Literatur für eine Ablösung der Orientierung durch Religion und durch Moralphilosophie. Mit der Religion wird die Einheitlichkeit des Weltbildes verabschiedet. Aber auch eine Moralphilosophie im Sinne Kants, die damit rechnet, dass der Mensch durch die Reinigung der Antriebsstrukturen seines freien Willens moralkompetent wird, hält Rorty für illusorisch.

Worin bestehen nun die Chancen der Literatur? Rorty wendet sich mit seinen Beispielen von Joyce und Proust ganz der erzählenden Literatur zu. Literatur schenke dem Leser Augenblicke des Verstehens, Erhebung der Gefühle, intime Identifikationen und deren Auflösung, zugleich aber auch Selbstdiagnosen und Selbstzweifel. Ihre destruktive Rolle sei dominant, aber sie enthalte subdominante Orientierungen, die sich für Momente herauslösen ließen, freilich nicht so, dass ein gleichbleibendes moralisches Konzept daraus entstünde.

Wenn auch nach Rorty die Literatur vorübergehende Potentiale der Orientierung enthält, die sich vor allem auf die Rücknahme von Selbstbezogenheit beziehen, also auf ein rudimentäres Programm der Willensreinigung, wie sie bei Kant in entfalteter Gestalt erscheint, ist sie doch der bleibenden Ungewissheit über das Gute und Richtige unterworfen. So stehen sich weiterhin die literarische Produktion von Ungewissheit und das Bedürfnis nach Gewissheit gegenüber. 


\section{Literarische Produktion von Ungewissheit und das Bedürfnis nach Gervissheit in der Begründung der Moral ${ }^{13}$}

Literarische Produktion von Ungewissheit erschüttert falsche oder scheinbare Gewissheiten. Auf der anderen Seite befriedigt sie die Erwartung von neuer Gewissheit nicht. Insofern tun sich manche schwer mit Literatur, die keine heile Welt heraufbeschwört, es sei denn mit ironischem Unterton. Darin kann ihnen die Literatur nicht helfen, aber wie ist es mit Moral und Religion? Nur zeitweise schien die Literatur sie zu beerben, heute spricht man von einem Boom der Ethik und von einer Wiederkehr des Religiösen.

Zunächst einmal ist festzuhalten, dass Ungewissheit auch als Grenze des rational Zugänglichen erscheint und dass diese Grenze mit literarischer Kompetenz aufgezeigt werden kann. Sie verweist aber damit zugleich auf einen tieferen $\mathrm{Zu}-$ sammenhang, in welchem die Ungewissheit zu einem allgemeinen Gefühl existentieller Ungesichertheit wird.

Aber gibt es nicht zwei Ebenen der Gewissheit: die Ebene der Wissenschaft und die Ebene der Weisheit? Als Beispiel für die Auseinandersetzung um zwei unterschiedliche Gewissheitsebenen kann man Blaise Pascals Gedanken heranziehen (vgl. Zwierlein 1996). Pascal erläutert die Differenz zwischen évidence und certitude. Evidenz meint hier nicht die gesicherte wissenschaftliche Erkenntnis, wie sie z. B. in einer ,evidenzbasierten Medizin' angestrebt ist. Das wäre für Pascal eher das Modell einer cartesianischen Gewissheit im Sinne von certitude, die der esprit de géométrie anstrebt. Evidenz ist für Pascal dagegen die Sicherheit der Urteilskraft, wie sie von Kant im Modell des ästhetischen Urteils beschrieben wird. ${ }^{14}$ Pascal meint mit ,Evidenz' das, was in der Herausbildung von Erfahrenheit (aufgrund von gelebter Erfahrung) und in mitmenschlicher Kommunikation als überzeugende Einsicht dem Menschen ,aufgeht' (vgl. Rombach 1987). Dies wiederum nennt Ricœur „Überzeugung" und er stellt eine Dialektik zwischen Überzeugung und Vernunft so dar, dass sie sich wechselseitig korrigieren und unterstützen können (vgl. Ricœur 1996).

Bei Kant erscheinen Urteilskraft und ethische Vernunft als unterschiedliche Ansätze. Während bekanntlich die Autonomie und der kategorische Imperativ auf dem „Faktum der Vernunft“ aufruhen und von daher begründet werden, ist das ästhetische, historische und politische Urteil einer Schulung der Erfahrung überantwortet. Die Erfahrungssicherheit, die aus sensibler Wahrnehmung, Erlebnis, Begegnung und deren Wiederholung bzw. Repräsentation in der Erzählung erwächst und auf die wir uns am Anfang dieser Ausführungen bezogen haben, ist eine andere als die Begründungssicherheit, die sich auf logische Kohärenz stützt:

13 Vgl. Mieth (2007a).

14 Kants Beispiele sind in seiner Kritik der Urteilskraft das historische, das künstlerische und das politische Urteil (Kant 2001). Paul Ricœur hat im Anschluß an Hannah Ahrendt diesen Ansatz im Hinblick auf das ethische Urteil weiter geführt (vgl. Ricœur 1995: 143-62). 
Ethik ist [...] kein Monolith, sie besteht vielmehr aus einer Pluralität von Ansätzen, Ansprüchen, Perspektiven, Begründungsformen und begrifflichen Angeboten, die sich in der Geschichte der Ethik herausgebildet haben und im Kontakt zu Fachwissenschaften weiter ausdifferenzieren. (Berendes 2007: 13)

Berendes spricht von einer ,streitlustigen Pluralität [...], ohne jedoch beliebig zu sein“, und er sieht „die Anforderung, sich in dieser Pluralität zu orientieren und zu positionieren" (Berendes 2007), an die Fachwissenschaftler, die sich in einem ethisch durchwachsenen Gelände bewegen. Sie können dann nicht einfach auf ihre „Moral“ zurückgreifen oder alle Moral ausblenden. Erst die akademische Ethik, die mit dem Zweifel an der eigenen Moral beginnt, bevor sie als normative Ethik Moral begründet bzw. als Strebensethik in Moral berät, ist im Hinblick auf moralische Vorurteile überprüfbar. Ein Motiv für meine eigene langjährige Beschäftigung mit narrativer Ethik war die Erfahrung eines Literaturstudiums, in welchem einerseits ästhetisch außerordentlich differenziert (und plural) bewertet wurde, in den ethischen Implikationen aber Bewunderungen und Abschätzigkeiten vorkamen, die als solche nicht auf ihre Vorurteile hin reflektiert wurden. Dies bezog sich z. B. auf die ästhetische Bewunderung und moralische Verwerfung von Gottfried von Straßburgs Tristan und Isolde.

Gewiss trifft man heute in dieser Hinsicht auf komplexere Bewertungen, die gleichsam von der aktuellen Pluralität gelebter Moralität angesteckt sind. Sind sie aber deshalb auch auf kundige Weise differenziert? Oder suchen sie eine bequeme Verweigerung reflektierter Ethik, weil sie diese für eine Sache individueller Einschätzung halten, die man nicht wissenschaftlich diskutieren kann? Dies entspricht dann umgekehrt der Abwehrhaltung derer, die ihren Lesegenuss lieber von literaturwissenschaftlichen Expertisen fern halten, weil sie deren Unbeliebigkeit und ständigem Paradigmenwechsel nicht trauen. Jedenfalls ist für eine narrative Ethik, die sich bereits auf eine plurale internationale Diskussion in Philosophie und Theologie stützen kann, zu beobachten, dass sie unter den erzähltheoretischen Handlungsmodellen noch keinen Platz gefunden hat. ${ }^{15}$

Konkrete Ethik, die sich aus der Abstraktheit rein theoretischer Entwürfe herauswagt, bedarf ihrerseits der innerwissenschaftlichen Kommunikation. Sie kann in den literaturwissenschaftlichen Bereichen nur dann präsent sein, wenn sie sich mit den analytischen Mitteln der Literaturwissenschaften (und ihren Konflikten) sowie ihren oft ironischen Darstellungsformen auskennt.

Gemeinsamer Gegenstand einer ethisch-literarischen Kommunikation ist zunächst ,das Ethische', nicht die Ethik. Gemeint sind damit handlungsrelevante Phänomene, die sich als solche nicht nur um ,wahr und ,falsch' (im Sinne von

15 Vgl. Martinez/Scheffel (2005: 145-59). Dort trifft man auf,Ethik' oder ,Moral' auch nicht im Begriffsregister. Freilich werden „Implikationen“ definiert, und zwar als: „Lediglich implizit oder unbestimmt gegebene, im Leseakt zu rekonstruierende Tatsachen der erzählten Welt. $\mathrm{Zu}$ unterscheiden sind analytische, pragmatische und literarisch-konventionelle Implikationen“ (189). Kommt das Ethische nur als das „Pragmatische“ vor? Auch bei Mieke Bals Narratology (1997) beschränkt sich die Beziehung zur Ethik auf Kulturanthropologie. 
,unwahr'), sondern auch um ,gut und schlecht', ,richtig und falsch' (im Sinne von ,unrichtig') bemühen. Diese Phänomene sind nicht Randerscheinungen des literarischen Bemühens, sondern sie gehören zu dem unbeliebigen Aufbau der Figuren und zu ihren Handlungsmöglichkeiten hinzu. Sie sind Bestandteile von zureichenden Motivationen, durch welche die Figuren und die Ereignisse plausibel werden. Das ,Ethische', das sich in diesem Sinne als erzählerisch uneindeutige (nicht etwa unbestimmte) Implikation des Erzählens erweist, kann Gegenstand eines gemeinsamen Entdeckungszusammenhanges von Literaturanalyse und Ethik sein. Die kohärente Begründung ästhetischer und ethischer Einsichten kann dann noch immer fruchtbar divergieren.

Wie wir gesehen haben, hat Literatur oft eine verunsichernde, dekonstruierende Rolle im Kontext moralischer Abwägungen. Dies ist auch dadurch bedingt, dass sie an absoluter Individualität interessiert ist, die sozusagen einen Grenzbegriff für das moralische Urteil darstellt. Wenn man versucht, einen absolut individuellen Casus unter die Allgemeinheit eines Urteils zu fassen, muss man entweder den Casus verurteilen oder das Urteil fallen lassen bzw. es zumindest erweitern oder verändern (wie es z. B. teilweise im kasuistischen Recht geschieht). Diese Alternativen stellen aber die Ethik vor Schwierigkeiten. Jean-Pierre Wils hat diese Schwierigkeiten als Ethiker mit einer moralischen Analyse der ,Nachsicht' aufzufangen versucht (vgl. Wils 2006). Diese stünde in der Tradition der aristotelischen Epikie, d. h. der Auslegung eines Maßstabes unter Berücksichtigung von Handlungsvoraussetzungen, Handlungsumständen und einer sinnvollen Auslegung von Regeln. Verstehen als Voraussetzung des Urteils? „Alles verstehen, heißt alles verzeihen“, besagt das bekannte französische Sprichwort. Dahinter mag eine tiefere Wahrheit stehen, aber das Verstehen hat noch eine andere Konnotation: es kann eine Tür zu einem richtigen Begründungsdiskurs sein, der mehr auf die Motive als auf diskursiven Argumente achtet. In der Tat scheint es mir ein Kennzeichen hermeneutischer Ethik zu sein, dass sie Verstehen und Begründen aufeinander bezieht. Wils versucht zu zeigen, dass Begründen ohne Verstehen nicht plausibel ist und dass Verstehen immer wieder das Interesse an kohärenter Begründung weckt.

Indem Literatur auf Modelle, zitierbare Kurzformen eines ethisch relevanten Musters, hin befragbar wird, in welchen sich vollzogene, aber auch versagte und gescheiterte Handlungsmöglichkeiten zeigen, fördert sie das Verstehen, das moralischen Evaluationen den Weg ebnen kann, ohne sich selbst für diesen Weg vereinnahmen zu lassen.

\section{Literatur}

Bal, Mieke (1997) Narratology: Introduction to the Theory of Narrative. 2. Aufl. Toronto: Univ. of Toronto Press.

Bay, Michael (2005) The Island (Film). Warner Bros. Pictures. 
Beck, Roman (2013) Transparenz in der biomedizinischen Forschung. Tübinger Studien zur Ethik. Bd. 1. Tübingen: Francke.

Berendes, Jochen (2007) „Einleitung“. Autonomie durch Verantwortung: Impulse für die Ethik in den Wissenschaften. Hg. Jochen Berendes. Paderborn: mentis. 7-19.

Bloom, Harold (1991) Die Heiligen Wabrheiten stürzen: Dichtung und Glaube von der Bibel bis zur Gegenwart. Frankfurt a. M.: Suhrkamp.

Blumenberg, Hans (1980) Der Prozess der theoretischen Neugierde. 2. Aufl. Frankfurt a. M.: Suhrkamp.

Booth, Wayne (1974) Die Rhetorik der Erzäblkunst. 2 Bde. Heidelberg: Quelle \& Meyer. Engl. Erstauflage (1961) The rhetoric of fiction. Chicago: Univ. of Chicago Press.

Brinkmann, Richard (1969) Theodor Fontane: Über die Verbindlichkeit des Unverbindlichen. Tübingen: Niemeyer.

Coetzee, John M. (2003) Elizabeth Costello: Eight Lessons. London: Secker \& Warburg.

Fiveson, Robert S. (1979) The Clonus Horror (Film). Group 1 International Distribution Organization Ltd.

Gadamer, Hans-Georg (1986) Wahrheit und Methode: Grundzüge einer philosophischen Hermeneutik. 5. Aufl. Tübingen: Mohr.

Genette, Gérard (1998) Die Erzäblung. München: Fink.

Goretta, Claude (1977) La dentellière/Die Spitzenklöpplerin (Film). Jupiter Communications.

Gottfried von Straßburg (2008) Tristan und Isolde. Übers. aus dem Mittelhochdt. Hermann Kurtz. München: Beck.

Haker, Hille (1999) Moralische Identität: Literarische Lebensgeschichten als Medium ethischer Reflexion. Tübingen: Francke.

- (2001) „Wahlverwandtschaften - Liebe, Sexualität und Fortpflanzung im Zeitalter der Reproduktionsmedizin“. Interdisziplinäre Ethik: Grundlagen, Methoden, Bereiche. Hgg. Adrian Holderegger und Jean-Pierre Wils. Freiburg i. Üe.: Universitätsverlag. 213-42.

- (2003) „Ban Graven Images - Literatur als Medium ethischer Reflexion“. Literatur ohne Moral. Hg. Christof Mandry. Münster: LIT. 67-88.

Haug, Walter (2001) „Das Böse und die Moral. Erzählen unter dem Aspekt einer narrativen Ethik“. Interdisziplinäre Ethik: Grundlagen, Methoden, Bereiche. Hgg. Adrian Holderegger und Jean-Pierre Wils. Freiburg i. Üe.: Universitätsverlag. 243-68.

Herms, Eilert (2008) Politik und Recht im Pluralismus. Tübingen: Mohr Siebeck.

Honneth, Axel (2012) Kampf um Anerkennung: zur moralischen Grammatik sozialer Konflikte. 7. Aufl. Frankfurt a. M.: Suhrkamp.

Ishiguro, Kazuo (2005) Never Let Me Go. London: Faber \& Faber. Dt. Ausgabe (2006) Alles, was wir geben mussten. Übers. Barbara Schaden. München: btb Verlag. 
Kant, Immanuel (2001) Kritik der Urteilskraft. Hg. Heiner F. Klemme. Hamburg: Meiner.

Krämer, Hans (1992) Integrative Ethik. Frankfurt a. M.: Suhrkamp.

Lainé, Pascal (1974) La dentellière. Paris: Gallimard. Dt. Ausgabe (1979) Die Spitzenklöpplerin. Übers. Eva Schewe. Reinbek: Rowohlt.

Lem, Stanisław (1998) Sterntagebücher. Übers. Caesar Rymarowicz. 16. Aufl. Frankfurt a. M.: Suhrkamp.

Martínez, Matías, und Michael Scheffel (2005) Einführung in die Erzäbltheorie. 6. Aufl. München: Beck.

Mieth, Dietmar (1983) Dichtung, Glaube und Moral. Studien zur Begründung einer Narrativen Ethik [1976]. 2. Aufl. Tübinger Theologische Studien 7. Mainz: Grünewald.

- (1985) „Ethos und Religion in der Science Fiction. Am Beispiel von Texten von Stanislaw Lem und Ursula Le Guin". Aspekte der Science Fiction in Ost und West. Hgg. Rolf-Dieter Kluge und Rolf Kellner. Tübingen: Verl. Science \& Fiction. 123-33.

- (1999) Moral und Erfahrung [1977]. 4. Aufl. Beiträge zur theologisch-ethischen Hermeneutik. Freiburg i. Üe.: Universitätsverlag.

- (2000a) Erzäblen und Moral: Narrativität im Spannungsfeld von Ethik und Ästhetik. Tübingen: Attempto.

- (2000b) „Ethik und Heuchelei am Beispiel der Biopolitik“. Ethik und Heuchelei. Hg. Eva Ruhnau. Köln: DuMont. 134-48.

- (2001) Die Diktatur der Gene. Freiburg i. Br.: Herder.

- (2002) Was wollen wir können? Ethik im Zeitalter der Biotechnik. Freiburg i. Br.: Herder.

- (2006) „Gewalt im Film und das Spiel der Ästhetik mit der Ethik“. Etbik und Ästhetik der Gewalt. Hgg. Julia Dietrich und Uta Müller-Koch. Paderborn: Mentis. 79-100.

- (2007a) „Insecuritas humana. Ungewissheit in Literatur, Ethik und Religion“. Impulse und Resonanzen: Tübinger mediävistische Beiträge zum 80. Geburtstag von Walter Haug. Tübingen: Niemeyer. 5-17.

- (2007b) „Literaturethik als narrative Ethik“. Narrative Ethik: Das Gute und das Böse erzäblen. Deutsche Zeitschrift für Philosophie. Sonderbd. 17. Hg. Joisten Karen. Berlin: Akademie. 215-34.

- (2009) „Science under the Spell of Prejudice. The Example of Biosciences“. Handbook of Prejudice. Hgg. Anton Pelinka, Karin Bischof, und Karin Stögner. New York: Cambria Press. 345-74.

Munro, Alice (2006) Der Traum meiner Mutter: Erzäblungen. Übers. Heidi Zerning. 2. Aufl. Frankfurt a. M.: Fischer.

Musil, Robert (1952) „Tonka [1922]“. Drei Frauen. Hamburg: Rowohlt Verlag. 57106.

Niccol, Andrew (1997) Gattaca (Film). Columbia Pictures. 
Nünning, Ansgar (1998) „Unreliable Narration zur Einführung: Grundzüge einer kognitiv-narratologischen Theorie und Analyse unglaubwürdigen Erzählens“. Unreliable Narration: Studien zur Theorie und Praxis unglaubwürdigen Erzäblens in der englischsprachigen Erzäblliteratur. Hgg. Ansgar Nünning, Carola Surkamp und Bruno Zerweck. Trier: WVT. 3-40.

Planck, Max (1959) Vorträge und Erinnerungen. Darmstadt: Wiss. Buchgesellschaft.

Ricœur, Paul (1987) „Narrative Identität“. Heidelberger Jabrbücher 31. Heidelberg/Berlin: Springer. 57-67.

- (1988-91) Zeit und Erzäblung. 3 Bde. München: Fink.

- (1995) Le juste. Paris: Éd. Esprit.

- (1996) Das Selbst als ein Anderer. München: Fink.

Romanek, Mark (2010) Never Let Me Go (Film). Fox Searchlight Pictures.

Rombach, Heinrich (1987) Strukturanthropologie. München: Alber.

Rorty, Richard (2003) „Der Roman als Mittel zur Erlösung aus der Selbstbezogenheit". Dimensionen ästhetischer Erfahrung. Hgg. Joachim Küpper und Christoph Menke. Frankfurt a. M.: Suhrkamp. 49-66.

Roth, Philip (2002) Der menschliche Makel. Übers. Dirk van Gunsteren. München/ Wien: Hanser.

Ruhnau, Eva (2000) Hg. Etbik und Heuchelei. Hg. vom Forschungszentrum Jülich. Köln: DuMont.

Schlink, Bernhard (1995) Der Vorleser. Zürich: Diogenes.

Schübel, Rolf (2003) Blueprint (Film). United International Pictures.

Taylor, Charles (1996) Quellen des Selbst: Die Entstebung der neuzeitlichen Identität. Frankfurt a. M.: Suhrkamp.

Tongeren, Paul von (2001) „Über die (Un-)Möglichkeit der Vergebung“. Interdisziplinäre Ethik: Grundlagen, Methoden, Bereiche. Hgg. Adrian Holderegger und Jean-Pierre Wils. Freiburg i. Üe.: Universitätsverlag. 288-398.

Twain, Mark (2008) The Adventures of Huckleberry Finn [1884]. London: Puffin.

Wils, Jean-Pierre (2006) Nachsicht: Studien zu einer ethisch-hermeneutischen Basiskategorie. Paderborn: Schöningh.

Zwierlein, Eduard (1996) Blaise Pascal zur Einfübrung. Hamburg: Junius Verlag. 